\title{
Longitudinal magnetization dynamics in Heisenberg magnets: Spin Green functions approach
}

\author{
(Review Article) \\ V.N. Krivoruchko \\ Donetsk Institute for Physics and Engineering the National Academy of Sciences of Ukraine \\ 46 Nauki Avenue, Kyiv 03028, Ukraine \\ E-mail: krivoruc@gmail.com
}

Received March 29, 2017, published online September 25, 2017

\begin{abstract}
In spite of the fact that dynamical properties of magnets have been extensively studied over the past years, the longitudinal magnetization dynamics is still much less understood than transverse one even in the equilibrium state of a system. In this paper, we give a review of existing, based on quantum-mechanical approach, theoretical descriptions of the longitudinal magnetization dynamics for ferro-, ferri- and antiferromagnetic dielectrics. The aim is to reveal specific features of this type of magnetization vibrations under description a system within the framework of one of the basic model theory of magnetism — the Heisenberg model. Related experimental investigations as well as open questions are also briefly discussed. We hope that understanding of the longitudinal magnetization dynamics distinctive features in the equilibrium state have to be a reference point for a theory uncovering the physical mechanisms that govern ultrafast spin dynamics after femtosecond laser pulse demagnetization when a system is far beyond an equilibrium state.
\end{abstract}

PACS: 75.78.-n Magnetization dynamics;

76.50.+g Ferromagnetic, antiferromagnetic, and ferrimagnetic resonances; spin-wave resonance;

75.78.Jp Ultrafast magnetization dynamics and switching.

Keywords: longitudinal magnetization dynamics, spin Green functions, Heisenberg magnets.

\section{Contents}

1. Introduction 1565

2. Longitudinal spin dynamics. Spin Green functions approach 1566

3. Ferromagnet 1567

4. Ferrimagnet 1569

5. Antiferromagnet 1572

6. Concluding remarks 1572

References 1573

\section{Introduction}

Ultrafast magnetization dynamics, i.e., spins dynamics on sub-picosecond (0.01-1 ps) or picosecond (10-100 ps) time scale $\left(1 \mathrm{ps}=10^{-12} \mathrm{~s}\right)$ is an important research direction in modern magnetism. This new research field gained widespread attention by the seminal observation of ultrafast laser-induced demagnetization of metallic Ni [1], the discovery of all-optical helicity dependent magnetic switching [2], the demonstration of toggle switching in the metallic ferrimagnet GdFeCo [3,4], and the recent discovery of alloptical switching in a broad class of metallic materials $[5,6]$.
These experiments revealed that excitation of a magnet system by intense femtosecond laser pulses causes a collapse of magnetic order much faster than any characteristic time (energy) scale of spin-spin interactions known at the time. Despite experimental progress, microscopic understanding of the ultrafast magnetization dynamics still remains an open question. At the same time, uncovering the physical mechanisms that govern ultrafast spin behavior is crucial for designing the next generation magnetic recording media and spin-based electronics. The state of the art of the ultrafast spin dynamics and its prospects can be found by the reader in the reviews [7-11]. 
At present, it is commonly accepted that the short time scale of a laser pulse and high temperatures following the excitation lead to processes when the longitudinal magnetization dynamics becomes pronounced [12-14]. In spite of the progress in phenomenological modeling the magnetic moment evolution at high temperatures during a time scale approaching femtoseconds [15-21], a number of questions still remain open. What is a magnetic configuration of a system just after ultrafast laser-induced heating? What is a local (quasi-) equilibrium state that the system acquires after the first sub-picosecond of ultrafast magnetization switching? Can the magnetic dynamics be treated as a quasi-static evolution if the system is in out-of-equilibrium state? What are the roles of various spin-resonance modes in these processes? Giving only these as examples. Naturally, to achieve a theoretical explanation of ultrafast magnetization dynamics, the microscopic consideration based on quantum-mechanical theory is indispensable. Any model claiming of correct description of ultrafast magnetization dynamics in the limit of a long-time evolution should reproduce longitudinal dynamics of the system in equilibrium state. Surprisingly, in spite of the fact that different aspects of magnetic system properties have been studied quite extensively over past years, rather little attention has been paid to the longitudinal magnetization dynamics. This type of magnetization excitations is still less well understood even for an equilibrium state of a magnetic system.

One of the basic microscopic model of magneto-dielectrics is the Heisenberg model [22]. In this paper, we give a brief review of the existing microscopic descriptions of the longitudinal magnetization dynamics in ferro-, ferriand antiferromagnets within the framework of this model. The paper is organized as follows. We begin by briefly reminding the key equations describing the magnetic system's linear response within the spin Green functions approach. Based on the results of earlier works of different authors, it will be shown that the system's longitudinal dynamic susceptibility $\chi^{\mathrm{zZ}}(\mathbf{q}, \omega)$ reduces to a calculation of the (sublattice) longitudinal correlation functions (the spin Green functions, GFs) $G_{i j}^{z Z}\left(\mathbf{q}, i \omega_{n}\right)$. A diagrammatic representation for the spin GFs as well as related distinction between the system's isothermal and isolated susceptibility tensors are briefly discussed here, too. The results obtained for the Heisenberg ferromagnet are expounded in Sec. 3. Investigations involving strongly renormalized spin-wave excitations existing at high temperatures confirm the multispin-wave nature of longitudinal spin dynamics in a ferromagnet suggested in the early studies of the model. Sections 4 and 5 are devoted to the results obtained for the Heisenberg ferrimagnets and antiferromagnets, respectively. Multisublattice nature of the system's magnetic structure reveals in the longitudinal magnetization precession through a few additional channels of multi-spin-wave creation/annihilation processes. The paper is concluded with some general remarks on further progress in the field.

\section{Longitudinal spin dynamics. Spin Green functions approach}

The Heisenberg model is a statistical mechanical model, in which the spins of the magnetic systems are treated quantum mechanically [22]. This model describes magnetic dielectrics which magnetism of ions in ith sites can be described by spin operator $\mathbf{S}_{i}=\left(S_{i}^{X}, S_{i}^{y}, S_{i}^{z}\right)$, and the interaction between spins is of the so-called exchange type: $J_{i j} \mathbf{S}_{i} \mathbf{S}_{j}$. Depending on a sign of the exchange interaction $J_{i j}=J\left(\mathbf{r}_{i}-\mathbf{r}_{j}\right)$ parallel (ferromagnetic) or antiparallel (antiferromagnetic or ferrimagnetic) spins states is realized below the critical temperature.

Within the microscopic approach (the spin Green function method [23-25]) description of a system's magnetization dynamics implies a calculation of the dynamic susceptibility tensor $\chi(\mathbf{q}, \omega)$ and investigation its properties as function of frequency $\omega$ and momentum q. This, in turn, is reduced to calculations of the retarded spin Green functions: $\chi(\mathbf{q}, \omega) \rightarrow G^{(R)}(\mathbf{q}, \omega)$. In particular, to find the system's longitudinal susceptibility $\chi^{z z}(\mathbf{q}, \omega)$ one should find the retarded longitudinal spin GF $G_{\text {tot }}^{\mathrm{zZ}(R)}(\mathbf{q}, \omega)$

$$
\chi^{\mathrm{zZ}}(\mathbf{q}, \omega)=\left\langle\left\langle\hat{T} M_{\text {tot }}^{\mathrm{Z}}(t) \mid M_{\text {tot }}^{\mathrm{z}}(0)\right\rangle\right\rangle_{\mathbf{q}, \omega}=-\frac{\mu_{B}^{2}}{v_{0}} G_{\text {tot }}^{z z(R)}(\mathbf{q}, \omega)
$$

Here $M_{\text {tot }}^{Z}$ is a $Z$ component of a total magnetization $\mathbf{M}_{\text {tot }}=\mu_{B} \Sigma_{i} g_{i} \mathbf{S}_{i}, \mu_{B}$ is the Bohr magneton, $g_{i}$ stands for the $g$ factor of the ith sublattice and $v_{0}$ stands for the volume of a primitive magnetic cell. The symbol $\left\langle<\ldots>_{\mathbf{q}, \omega}\right.$ denotes the Fourier transform of the trace of $\rho(\ldots)$ with $\rho=\exp (-\beta \hat{H}) / \operatorname{Sp}(\exp (-\beta \hat{H})) ; \hat{T}$ is the time-ordering operator; $\beta^{-1}=T$ is the temperature and $\hat{H}$ is the Hamiltonian. There are theorems proving that the poles of the retarded GFs correspond to the natural frequencies of magnetization excitations that are (i) transverse magnetization oscillations of the spins or ordinary spin-waves, and (ii) longitudinal spin oscillations. By turn, the retarded GFs of the system can be restored from the temperature GFs $G\left(\mathbf{q}, i \omega_{n}\right)$ by analytic continuation from the Matsubara frequencies $i \omega_{n}=i 2 \pi n T(n=0, \pm 1, \pm 2, \ldots)$ onto the real frequency axis $i \omega_{n} \rightarrow \omega+i \delta(\delta \rightarrow 0)$ (for more details, see, e.g., Refs. 23-25).

For a ferromagnetic system, the total GF $G_{\text {tot }}^{z z(R)}(\mathbf{q}, \omega)$ reads

$$
G_{\text {tot }}^{z Z}\left(\mathbf{q}, i \omega_{n}\right)=G_{\text {tot }}^{z Z}(q)=g^{2}\left\langle\left\langle\hat{T}\left(S_{\mathbf{f}}^{Z}-\left\langle S^{Z}\right\rangle\right) \mid\left(S_{\mathbf{g}}^{Z}-\left\langle S^{Z}\right\rangle\right)\right\rangle\right\rangle_{\mathbf{q}, \omega} .
$$

Here and below we use the notation $q=\left\{\mathbf{q}, i \omega_{n}\right\}$, where $\mathbf{q}$ stands for the momentum and the Matsubara frequency $i \omega_{n}$.

In the case of a system with two antiparallel sublattices, the total GF $G_{\text {tot }}^{z Z(R)}(\mathbf{q}, \omega)$ can be reduced to four sublattice longitudinal GFs $G_{i j}^{Z Z}\left(\mathbf{q}, i \omega_{n}\right)(i, j=1,2)$ as follows: 


$$
\begin{gathered}
G_{\text {tot }}^{Z Z}(q)=\left\langle\left\langle\hat{T}\left(g_{1} \delta S_{1}^{Z}-g_{2} \delta S_{2}^{Z}\right) \mid\left(g_{1} \delta S_{1}^{Z}-g_{2} \delta S_{2}^{Z}\right)\right\rangle\right\rangle= \\
=g_{1}^{2} G_{11}^{Z Z}(q)-g_{1} g_{2}\left[G_{12}^{Z Z}(q)+G_{21}^{Z Z}(q)\right]+g_{2}^{2} G_{22}^{Z Z}(q),
\end{gathered}
$$

where $\delta S_{i}^{Z} \equiv S_{i}^{Z}-\left\langle S_{i}{ }^{Z}\right\rangle$ and $G_{i j}^{Z Z}=\left\langle\left\langle\hat{T}_{\tau}\left(S_{i}^{Z}-\left\langle S_{i}^{Z}\right\rangle\right) \mid\left(S_{j}^{Z}-\left\langle S_{j}^{Z}\right\rangle\right)\right\rangle\right\rangle$. Thus, for a multisublattice system, calculation of the dynamic susceptibility $\chi^{z Z}(\mathbf{q}, \omega)$ reduces to calculation of the sublattice longitudinal GFs $G_{i j}^{z Z}\left(\mathbf{q}, i \omega_{n}\right)$.

To find the spin GFs it is convenient to use the diagrammatic technique for spin operators. General rules for constructing particular diagrams can be found, e.g., in Refs. 23-25. We note only here that there are two sorts of the $S^{z}$ vertices: (a) without attached Green's lines of transverse spin-waves and (b) with one incoming and one outgoing Green's lines. There is one sort of the $S^{-}=S^{x}-i S^{y}$ vertices with one outgoing Green's line, while two sorts of the $S^{+}=S^{x}+i S^{y}$ vertices are present, with one incoming Green's line or, with two incoming and one outgoing Green lines. All internal vertices are joined by lines of interaction $J_{i j}$.

To calculate the longitudinal spin GFs, we use the Larkin equation derived earlier in the framework of the diagrammatic technique for spin operators. Without dwelling into the details of summing up the diagram series (Refs. 23-25 contain technical details concerning the construction of the diagram technique for the Heisenberg magnet), we present here the final analytic results. One can show that the graph series for the $G^{Z Z}(q)$ functions of a ferromagnet can be presented analytically in the form

$$
G^{z Z}(q)=\Sigma^{z}(q)\left[1-J_{q}^{Z} \Sigma^{z}(q)\right]^{-1} .
$$

In terms of the diagrammatic technique, the quantity $\Sigma^{Z}(q)$ is called an irreducible (by the Larkin's method of isolating the irreducible diagrams) part. Note that the irreducibility is understood here in the sense that $\Sigma^{z}(q)$ is represented by the collection of all diagrams from the series for $G^{z Z}(q)$ that cannot be cut across one line of interaction $J_{\mathbf{q}}\left(J_{\mathbf{q}}\right.$ is the Fourier transform of the exchange interaction).

Within this diagrammatic technique, the graphical representation of the longitudinal susceptibility $\chi^{\text {ZZ }}(\mathbf{q}, \omega)$ contains two external $S^{z}$ vertices which could be classified into three types of diagrams. Namely, diagrams where: (i) all external $S^{Z}$ vertices are isolated, (ii) one external $S^{Z}$ vertex is isolated and another is connected by incoming and outgoing transverse Green's lines, and (iii) all external $S^{Z}$ vertices are connected by incoming and outgoing transverse spin-waves lines. Thus, the mathematical problem amounts to summing up all the diagrams describing these processes. A complexity of the problem arises from the fact that a commutator of two spin operators is not a $c$ number but an operator, too. Therefore, the series of the diagrams turns out to be rather complicated in comparison with Bose or Fermi operators.

In accordance with the diagram technique rules, the analytical expressions of the (i) and (ii) types diagrams for
$\Sigma^{Z}\left(\mathbf{q}, i \omega_{n}\right)$ will contain a singular discrete frequency part $\sim \delta \omega_{n, 0}$ [26] and, thus, will not depend on the thermodynamic time (here $\delta_{n, 0}$ is the Kronecker delta, being 0 if the two arguments are different and 1 if they are equal). In fact, we get here into touch with the difference between the isolated and isothermal susceptibilities of a system [27-29]. The isolated (the Kubo) susceptibility is defined for the case when a system is initially in thermal equilibrium and isolated. The isothermal susceptibility is defined for a system assumed to be in thermal equilibrium with thermostat in the presence of a time-independent external «force». In accordance with the general analysis of different susceptibilities [27-29] the distinction between them points to the nonergodicity of the system. Below we will be interested in the Kubo (or isolated) susceptibility of the system and discuss the related analytical expressions derived from the quantity $G_{\mathrm{tot}}^{Z Z}\left(\mathbf{q}, i \omega_{n}\right)$ by analytic continuation from the Matsubara frequencies onto a real axis $i \omega_{n} \rightarrow \omega+i \delta$ $(\delta \rightarrow 0)$.

Note here, that typically the irreducible parts $\Sigma_{i j}^{Z}(q)$ could be calculated by summing up the ladder diagrams with antiparallel lines. Such summing up corresponds to the so-called generalized random-phase approximation (RPA) and rather well describes the ground state and dynamics of a magnetic system (see, for example, Ref. 25). Note also here, that as it is known, the RPA results hold valid beyond the hydrodynamic regime, i.e., when the wave vector is larger than the inverse correlation length $\xi$ : $q>1 / \xi \sim\left(1-T / T_{C}\right)^{1 / 2}$, and, of course, beyond the critical region (here $T_{C}$ is the Curie temperature; for more details, see, e.g., Ref. 30).

\section{Ferromagnet}

An isotropic Heisenberg ferromagnet is described by the Hamiltonian

$$
H=-\frac{1}{2} \sum_{f, g} J_{f g} \mathbf{S}_{f} \mathbf{S}_{g}=-\frac{1}{2} \sum_{f, g} J_{f g}\left[\left(S_{f}^{+} S_{g}^{-}+S_{g}^{+} S_{f}^{-}\right)+S_{f}^{Z} S_{g}^{Z}\right] .
$$

Here the circular spin operators $S_{g}^{ \pm}$are defined as $S_{g}^{ \pm}=\left(S_{g}^{x} \pm i S_{g}^{y}\right) / \sqrt{2}$, and $J_{f g}(>0)$ stands for the exchange integral between localized spins in $f$ th and $g$ th sites.

In the pioneering paper [31] Vaks, Larkin and Pikin proposed original diagram technic for spin operators and studied magnetization dynamics in the Heisenberg ferromagnet. Using the standard perturbation approach (an expansion in the terms of $1 / z$, where $z$ is the first coordination lattice number) they showed that the transverse coherent precession of magnetization (elementary spin-wave excitations or magnons) survive in the long wavelength limit at all temperatures below the critical one $T_{C}$. For the longitudinal correlations, they obtained an expression of the form 


$$
G^{z z(0)}\left(\mathbf{q}, i \omega_{n}\right)=\frac{b^{\prime}}{1-\beta J_{\mathbf{q}} b^{\prime}} \delta_{n, 0}
$$

in the zeroth order of $1 / z$, and

$$
G^{z z}\left(\mathbf{q}, i \omega_{n}\right)=\frac{1}{N} \sum_{\mathbf{k}} \frac{n\left(\varepsilon_{\mathbf{k}}\right)-n\left(\varepsilon_{\mathbf{k}-\mathbf{q}}\right)}{\omega-\varepsilon_{\mathbf{k}}+\varepsilon_{\mathbf{k}-\mathbf{q}}+i \delta}
$$

in the first order of the terms of $1 / z$. Here $b^{\prime}$ is the first derivative of the function $b(z)=S B_{S}(z)$, and $B_{S}(z)$ stands for the Brillouin function, $\delta_{n, 0}=\delta_{\omega_{n}, 0}, \varepsilon_{\mathbf{k}}$ is the spin-wave energy and $n\left(\varepsilon_{k}\right)=\left[\exp \left(\varepsilon_{k} / T\right)-1\right]^{-1}$ is the Bose distribution function. Equation (6) represents the isothermal susceptibility of the system. Note that within this approximation its characterizes static fluctuations of the longitudinal spin components, which are characterized by the Brillouin functions derivatives. The isolated (the Kubo) susceptibility is described by expression (7).

Thus, in accordance with Eq. (7), the uniform susceptibility $\chi^{Z Z}(\mathbf{0}, \omega)=0$ for $\omega \neq 0$. This is a consequence of the total spin conservation law. Generally, in the noninteracting spin-wave approximation, the longitudinal spin excitations eventuate as virtual processes of coherent creation and annihilation of transverse spin-waves. For a ferromagnet, the longitudinal excitation frequency - a pole of the denominator in Eq. (7) - is $\omega(\mathbf{q})=\varepsilon_{\mathbf{k}}-\varepsilon_{\mathbf{k} \pm \mathbf{q}}$ (here the wave vector $\mathbf{k}$ is a variable). Also, the excitation processes are controlled by the occupation factor, determined through the Bose distribution function $n\left(\varepsilon_{\mathbf{k}}\right)$, which makes the spin-waves with $\mathbf{k} \sim 0$ to be dominant ones.

Note that this peculiarity of the longitudinal spin dynamics is a general one and is determined by specific quantum properties of the spin operators. Namely, the transverse operators $S^{+}$and $S^{-}$are operators of one spinwave creation (annihilation), and the commutator of these operators is the operator $S^{Z}$. As a result, the longitudinal vibrations of magnetization by external magnetic field, $\sim h_{z} S^{Z}$, may be realized only as virtual processes of coherent creation and annihilation of two transverse spin-waves: $h_{z} S^{Z} \rightarrow h_{z}\left(S^{+} S^{-}\right)$. For multisublattice magnets, the magnetization longitudinal dynamics can also be realized through virtual processes of two different transverse modes creation and annihilation (see below).

The results [31] were then confirmed and generalized in more elaborated studies based on a modern diagram technique for spin operators by Izymov et al. [32]. Authors summed up infinite series of diagrams for the irreducible part $\Sigma^{Z}(q)$ of the longitudinal GF (4) involving all distinct loops built from spin-wave propagators and describing the spin-wave interaction. In Fig. 1 one- and two-loop order diagrams for the irreducible part $\Sigma^{Z}(q)$ of the longitudinal spin GF (4) are shown. The diagrams representing the Kubo susceptibility are only shown explicitly. In the figure, two external vertices of the GFs are represented by vertices which are a hollow point with an incoming and outgoing

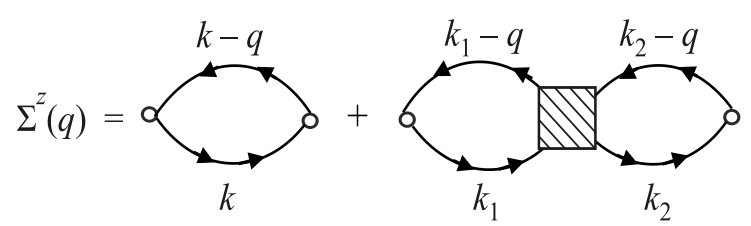

Fig. 1. The one- and two-loop order diagrams for irreducible part of the longitudinal spin GF. The diagrams describing the Kubo susceptibility are only shown explicitly. Here and in Fig. 3 external hollow vertices correspond to external $S^{Z}$ spin operators. Here and in Figs. 2-4, the solid lines represent the transverse spinwave GFs; the hatched squares represent graphically the effective four-point vertices.

Green lines. The hatched square here represents graphically the effective four-point scattering vertex. The corresponding analytical expression is

$$
\begin{gathered}
\Sigma^{Z}(q)=(\beta N)^{-1} \sum_{k} G(k) G(k-q)+ \\
+(\beta N)^{-2} \sum_{k, p} G\left(k_{1}\right) G\left(k_{2}-q\right) \Gamma\left(k_{1}, k_{2}-q \mid k_{2}, k_{2}+q\right) G\left(k_{2}-q\right) G\left(k_{2}\right),
\end{gathered}
$$

where $k=\left\{\mathbf{k}, i \omega_{n}\right\}$. Within this perturbation approach, the next step is a calculation of the four-point vertex $\Gamma\left(k_{1}, k_{2}-q \mid k_{2}, k_{2}+q\right)$ - the spin-wave scattering amplitude - presented graphically in Fig. 2 within the general RPA. Here the wavy line is a graphical representation of the interaction $J_{q}$. The graphical equation in Fig. 2 is expressed analytically as follows:

$$
\begin{gathered}
\Gamma\left(k_{1}, k_{2} \mid k_{1}-q, k_{2}+q\right)=J_{\mathbf{k}_{2}+\mathbf{q}}+J_{\mathbf{k}_{1}-\mathbf{q}}+ \\
+(\beta N)^{-1} \sum_{k_{3}} J_{\mathbf{k}_{3}+\mathbf{q}} G\left(k_{3}\right) G\left(k_{3}+q\right) \Gamma\left(k_{3}+q, k_{2} \mid k_{3}, k_{2}+q\right)+ \\
+J_{\mathbf{k}_{1}-\mathbf{q}}(\beta N)^{-1} \sum_{k_{3}} G\left(k_{3}+q\right) G\left(k_{3}\right) \Gamma\left(k_{3}+q, k_{2} \mid k_{3}, k_{2}+q\right) .
\end{gathered}
$$

This integral equation can be transformed into an algebraic one (for details, see Ref. 32).

After a simple but cumbersome algebra, in accordance with the results of [31], for a ferromagnet the longitudinal

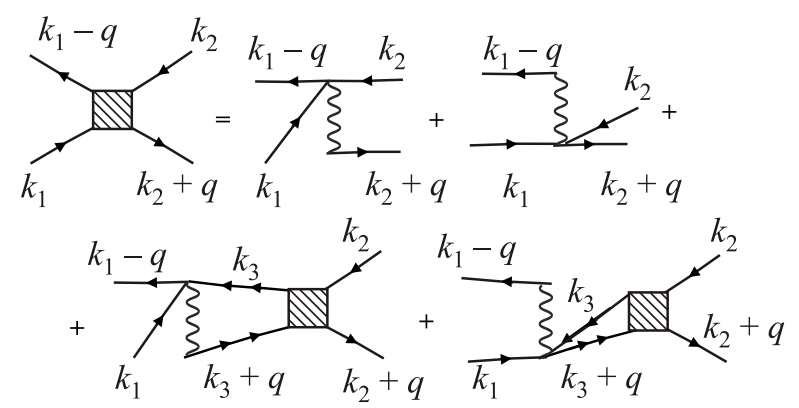

Fig. 2. Graphical representation the equation for the effective four-point vertex $\Gamma\left(k_{1}, k_{2} \mid k_{1}-q, k_{2}+q\right)$. Here and in Fig. 4 a wavy line corresponds to the interaction $J_{\mathbf{q}}$. 
excitations have been found at frequencies $\omega(\mathbf{q})$ which are typical for processes of creation and annihilation of spinwaves with energies $\varepsilon_{\mathbf{k}}$ and $\varepsilon_{\mathbf{k} \pm \mathbf{q}}$. Also, it was confirmed that the excitation processes are controlled by the occupation factor, determined through the Bose distribution function $n\left(\varepsilon_{\mathbf{k}}\right)$. However, beyond the formal similarity, there is a notable difference among two results due to a much more common character of the approximation used in [32]. The complete expression for $\chi^{Z Z}(\mathbf{q}, \omega)$ obtained in [32] describes a strong renormalization of the energy longitudinal spin fluctuations. Within the quadratic dispersion law for spinwave $\varepsilon_{\mathbf{q}}=\left\langle S^{z}\right\rangle J(a q)^{2}$ ( $a$ is the lattice spacing), the dispersion law of renormalized longitudinal wave excitations was found to be of linear wave vector dependence $\omega(q) \approx 0.43\left\langle S^{z}\right\rangle J(a q)$. I.e., in a ferromagnet the frequency of collective vibrations of the spin longitudinal component lie energetically above the transverse spin-waves frequency at the same temperature and wave vector. It was also shown that the dynamic structure factor $\omega^{-1} \operatorname{Im} \chi^{z Z}(\mathbf{q}, \omega)$ exhibits generally a three-peak structure including, first, two wide maxima at frequencies $\omega \sim \pm\left\langle S^{Z}\right\rangle J(a q)$ corresponding to damped longitudinal wave modes and, second, a sufficiently narrow and less intensive central peak. When approaching the Curie temperature, $T_{C}$, the intensity of the latter grows, and all three peaks form a broad distribution with the linearly $q$-dependent width.

These results give a natural explanation for seemingly conflicting experimental observations of two-peaked and single-peaked behavior, found in numerous inelastic neutron scattering studies of ferromagnetic materials. At the same time, the contradictory results of neutron studies of longitudinal fluctuations in ferromagnets motivated researchers to explore the longitudinal susceptibility of a ferromagnet beyond the model (1). Authors [33,34] investigated theoretically an isotropic ferromagnet with dipolar-dipolar interaction in different temperature regions. It was shown that dipole-dipole forces cannot be considered as a perturbation not only in the close vicinity of the Curie point but also at any temperature for low enough frequencies. The longitudinal susceptibility reveals the infrared discontinuity in the zero-frequency limit. In zero internal magnetic field, $H_{\text {int }}$, the susceptibility demonstrated the phenomenon of infrared divergence. At finite $H_{\text {int }}$ there exists a threshold for $\operatorname{Im} \chi^{Z Z}(\omega)$ at the minimal energy of two magnons creation. Therefore, one could expect an anomalous low-frequency behavior of the longitudinal spin susceptibility in weak external magnetic fields at any temperature below $T_{C}$. Authors [34] presented the experimental evidence that in the ideal isotropic ferromagnet dipolar forces would lead to the instability of the magnetically ordered state. They attributed the anomalous behavior of the dynamical longitudinal susceptibility observed in the ordered state of a nearly isotropic ferromagnet $\mathrm{CdCr}_{2} \mathrm{Se}_{4}$ to the influence of the dipole-dipole interaction and discuss the problem related to this feature.
Rudoy [35] studied the longitudinal dynamic susceptibility of easy axis anisotropic Heisenberg ferromagnet in the presence of a weak external magnetic field (supposed time dependent and spatially non-uniform) at low temperatures $T<<T_{C}$. Note that, at low temperatures, the spin system like a Heisenberg ferromagnet is very similar, but not identical, to a non-ideal gas of Bose particles. In condensed Bose or Fermi systems some collective motions like zero sound or plasma oscillation can arise under certain conditions. If this type of excitations could exist in a Heisenberg ferromagnet (the so-called «zero-magnon») it would appear as a singularity in the longitudinal dynamic susceptibility. In [35], linearized quantum equations of motion for the longitudinal components of the spin operators are employed to construct the dynamic RPA for the magnon collective GF. The one-parameter class of the bosonic representations of spin operators was used. The dispersion equation, describing the poles of the longitudinal dynamic susceptibility, is studied and it is shown that in the easy-axis anisotropic Heisenberg ferromagnet there are no long-wavelength excitations of the «zero-magnon» type.

\section{Ferrimagnet}

Dynamical properties of ferrimagnetic compounds substantially differ from those of ferromagnetic ones because of their multisublattice magnetic structure. Let us consider an isotropic model of a two-sublattice ferrimagnet when interaction inside the sublattices is small and can be neglected, and $g$ factors of the sublattices are equal, $g_{1}=g_{2}$. In absence of any external influences, the atomistic spin model is described purely by the exchange interaction, given by the Heisenberg Hamiltonian:

$$
H=\sum_{\mathbf{f}, \mathbf{g}} J_{\mathbf{f g}}\left[\frac{1}{2}\left(S_{\mathbf{l f}}^{+} S_{2 \mathbf{g}}^{-}+S_{2 \mathbf{g}}^{+} S_{1 \mathbf{f}}^{-}\right)+S_{1 \mathbf{f}}^{Z} S_{2 \mathbf{g}}^{Z}\right] .
$$

Pikin [36] studied the transverse magnetization dynamics in the model (10) and showed that in a such system, as in a ferromagnet, in the long wavelength limit the elementary spin-wave excitations survive at all temperatures below the critical one.

As was already shown in Sec. 2, for a two-sublattice system the calculation of the susceptibility $\chi^{z Z}(\mathbf{q}, \omega)$ is reduced to the calculation of four sublattice longitudinal GFs $G_{i j}^{Z Z}\left(\mathbf{q}, i \omega_{n}\right)$ of the form (3). As was shown in [36], within the zeroth order of a large interaction radius (the zeroth order of the parameter $1 / z$ ) longitudinal sublattice correlators are

$$
\begin{gathered}
G_{11}^{Z Z(0)}\left(\mathbf{q}, i \omega_{n}\right)=\frac{b_{1}^{\prime}}{1-\left(\beta J_{\mathbf{q}}\right)^{2} b_{1}^{\prime} b_{2}^{\prime}} \delta_{n, 0}, \\
G_{22}^{Z Z(0)}\left(\mathbf{q}, i \omega_{n}\right)=\frac{b_{2}^{\prime}}{1-\left(\beta J_{\mathbf{q}}\right)^{2} b_{1}^{\prime} b_{2}^{\prime}} \delta_{n, 0}, \\
G_{12}^{Z Z(0)}\left(\mathbf{q}, i \omega_{n}\right)=G_{21}^{Z z(0)}\left(\mathbf{q}, i \omega_{n}\right)=\frac{\beta J_{\mathbf{q}}^{Z} b_{1}^{\prime} b_{2}^{\prime}}{1-\left(\beta J_{\mathbf{q}}\right)^{2} b_{1}^{\prime} b_{2}^{\prime}} \delta_{n, 0} .
\end{gathered}
$$


As for a ferromagnet, within this approximation we deal with the isothermal susceptibility which describes static fluctuations of the longitudinal spin components.

To restore the dynamical characteristics, one needs to find the GFs $G_{i j}^{Z Z}(q)$ within a high order approximation. These calculations have been done just recently. In the report [37], based on the modern version diagram technique for spin operators, the analytical expression for the longitudinal susceptibility of a two-sublattice ferrimagnet has been obtained. Examples of the one- and two-loop order diagrams for the irreducible part $\Sigma_{11}^{Z}(q)$ of the longitudinal spin Green function $G_{11}^{Z Z}(q)$ are shown in Figs. 3(a) and (b), respectively. In the figures, the diagrams describing the Kubo susceptibility are shown only. Graphical representations for the irreducible parts $\Sigma_{12}^{Z}(q), \Sigma_{21}^{Z}(q)$, and $\Sigma_{22}^{Z}(q)$ possess a similar structure (for details, see Ref. 37). As in the case of a ferromagnet, in Fig. 3, two external vertices of the GFs are represented by vertices which are a hollow point with an incoming and outgoing Green lines. Other arrangements of external vertices do not exist. Here the hatched squares represent graphically two effective four-point scattering vertexes. Graphical representation the equation for effective four-point vertex (a) $\Gamma_{11,12}\left(k_{1}, k_{2} \mid k_{1}-q, k_{2}+q\right)$ and (b) $\Gamma_{22,21}\left(k_{1}, k_{2} \mid k_{1}-q, k_{2}+q\right)$ is shown in Fig. 4. Within this perturbation approach the longitudinal spin susceptibility reads as:

$$
\chi^{z Z}(\mathbf{q}, \omega)=-\left(\mu_{B}^{2} / v_{0}\right) \frac{N(\mathbf{q}, \omega)}{D(\mathbf{q}, \omega)},
$$

where [37]

$$
\begin{gathered}
N(q)=g_{1}^{2} \Sigma_{11}^{z}(q)- \\
-2 g_{1} g_{2}\left[\sum_{12}^{Z}(q)-J_{q} \sum_{12}^{z}(q) \sum_{21}^{z}(q)+\right. \\
\left.+J_{q} \sum_{11}^{z}(q) \sum_{22}^{Z}(q)\right]+g_{2}^{2} \sum_{22}^{Z}(q)
\end{gathered}
$$

(a)

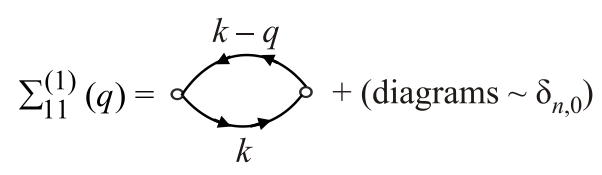

(b)

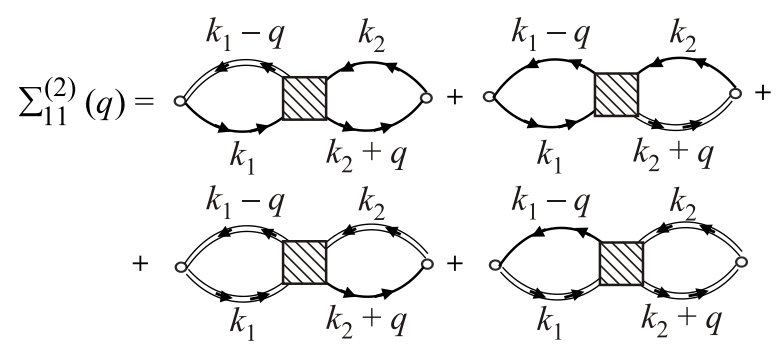

Fig. 3. The one-order (a) and two-loop orders (b) diagrams for irreducible parts of the longitudinal spin GFs. Only the diagrams describing the Kubo susceptibility are shown explicitly. Here and in Fig. 4 the solid line describes the transverse spin-wave GF $G_{11}(q)$; the double solid line describes the transverse spin-wave GF $G_{12}(q)$ or $G_{21}(q)$. (a)

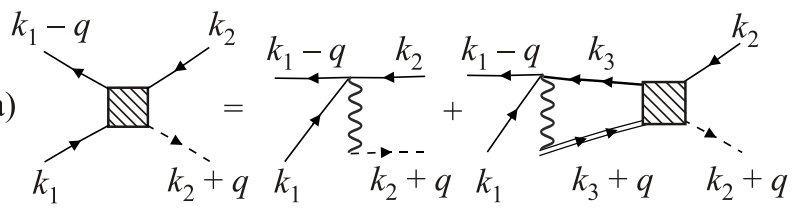

(b)

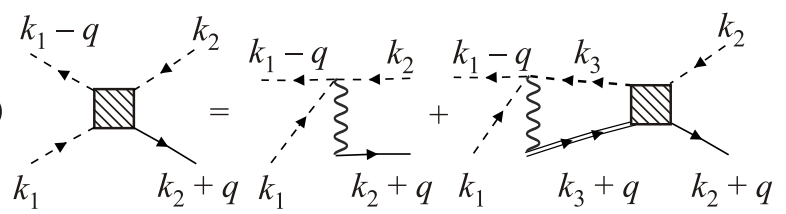

Fig. 4. Graphical representation the equation for the effective four-point vertex $\quad$ (a) $\Gamma_{11,12}\left(k_{1}, k_{2} \mid k_{1}-q, k_{2}+q\right)$ and (b) $\Gamma_{22,21}\left(k_{1}, k_{2} \mid k_{1}-q, k_{2}+q\right)$. Here the dashed line describes the transverse spin-wave $\mathrm{GF}_{22}(q)$.

and

$$
D(q)=\left[1-J_{\mathbf{q}} \Sigma_{12}^{Z}(q)\right]\left[1-J_{\mathbf{q}} \Sigma_{21}^{Z}(q)\right]-J_{\mathbf{q}}^{2} \Sigma_{11}^{Z}(q) \Sigma_{22}^{Z}(q) .
$$

In the one-loop order approximation, after summing up over the discrete Matsubara frequency analytical expressions for the functions $\Sigma_{12}^{Z}(q)$ and $\Sigma_{21}^{Z}(q)$ acquire a form

$$
\begin{gathered}
\Sigma_{12}^{Z}(q)=\Sigma_{21}^{Z}(q)= \\
=\frac{b_{1}^{2} b_{2}^{2}}{N} \sum_{\mathbf{p}} \frac{J_{\mathbf{p}}}{\varepsilon_{1 \mathbf{p}}+\varepsilon_{2 \mathbf{p}}} \frac{J_{\mathbf{p}-\mathbf{q}}}{\varepsilon_{1 \mathbf{p}-\mathbf{q}}+\varepsilon_{2 \mathbf{p}-\mathbf{q}}}\left\{\frac{n_{1}\left(\varepsilon_{1 \mathbf{p}}\right)-n_{1}\left(\varepsilon_{1 \mathbf{p}-\mathbf{q}}\right)}{i \omega_{q}-\varepsilon_{1 \mathbf{p}}+\varepsilon_{1 \mathbf{p}-\mathbf{q}}}+\right. \\
+\frac{n_{2}\left(\varepsilon_{2 \mathbf{p}-\mathbf{q}}\right)-n_{2}\left(\varepsilon_{2 \mathbf{p}}\right)}{i \omega_{q}+\varepsilon_{2 \mathbf{p}}-\varepsilon_{2 \mathbf{p}-\mathbf{q}}}+\frac{1+n_{1}\left(\varepsilon_{1 \mathbf{p}-\mathbf{q}}\right)+n_{2}\left(\varepsilon_{2 \mathbf{p}}\right)}{i \omega_{q}+\varepsilon_{2 \mathbf{p}}+\varepsilon_{1 \mathbf{p}-\mathbf{q}}}- \\
\left.-\frac{1+n_{1}\left(\varepsilon_{1 \mathbf{p}}\right)+n_{2}\left(\varepsilon_{2 \mathbf{p}-\mathbf{q}}\right)}{i \omega_{q}-\varepsilon_{1 \mathbf{p}}-\varepsilon_{2 \mathbf{p}-\mathbf{q}}}\right\} .
\end{gathered}
$$

The expressions for the irreducible parts $\Sigma_{11}^{Z}(q)$ and $\Sigma_{22}^{Z}(q)$ have the similar structure (see Ref. 37). Here within the long-wave approximation, $(a q)<<1$,

$$
\varepsilon_{1 \mathbf{q}}=D(a q)^{2}, \quad D=2 \frac{b_{1} b_{2}}{b_{1}-b_{2}} J_{0}
$$

is the energy of acoustic, and

$$
\varepsilon_{2 \mathbf{q}}=\left(b_{1}-b_{2}\right) J_{0}+D(a q)^{2},
$$

exchange spin-wave modes (in-phase and anti-phase sublattices magnetization precession, respectively).

As it follows from the analysis of the irreducible parts $\Sigma_{i j}^{Z}(q)$ [see, e.g., Eq. (12)], in a ferrimagnet, dynamics of longitudinal spin components is due to a few virtual processes of creation and annihilation of transverse excitations. Namely, the first channel - the first item in the right-hand-site brace of Eq. (12) - represents the processes of creation and annihilation of spin-waves with energies $\varepsilon_{1 \mathbf{p}}$ and $\varepsilon_{1 \mathbf{p} \pm \mathbf{q}}$ which correspond to in-phase precession of sublattices magnetization. This channel is controlled by the occupation factor of acoustic magnons, $n_{1}\left(\varepsilon_{1 \mathbf{p}}\right)$, which 
makes the spin-waves with $\mathbf{p} \sim 0$ to be dominant ones. A simple comparison reveals that this channel is a direct analog of the magnetization longitudinal dynamics in a ferromagnet found by Izumov et al. [32].

There is also the second channel - the second term in the right-hand-site of Eq. (12) — with characteristic excitation energy $\omega(\mathbf{q})=\varepsilon_{2 \mathbf{p}}-\varepsilon_{2 \mathbf{p} \pm \mathbf{q}}$. It corresponds to a virtual creation and annihilation of exchange spin-waves with anti-phase precession of sublattices magnetization. This channel is also controlled by the related occupation factor determined through the Bose distribution functions $n_{2}\left(\varepsilon_{2} \mathbf{p}\right)$, which makes exchange spin-waves with $\mathbf{p} \sim 0$ dominant in this way of longitudinal spin dynamics.

The last two items in the brace of Eq. (12) represent the third channel of longitudinal excitations. Namely, there is a two-spin-wave creation/annihilation process at frequency $\omega(\mathbf{q})=\varepsilon_{1} \mathbf{p}+\varepsilon_{2} \mathbf{p} \pm \mathbf{q}$ which corresponds to creation or annihilation of one acoustic and one exchange transverse modes. This channel remains in force even in the absence of thermal excitations, i.e., when $n_{1}\left(\varepsilon_{1 \mathbf{p}}\right) \sim 0$ and/or $n_{2}\left(\varepsilon_{2 \mathbf{p}}\right) \sim 0$. We note also some important new in comparison with the case of a ferromagnet feature of the $\chi^{z z}(\mathbf{q}, \omega)$. As it follows already from Eq. (12) [see last two items in Eq. (12)], for a ferrimagnetic system the uniform longitudinal susceptibility $\chi^{z Z}(\mathbf{0}, \omega)$ is nonzero, that reflects the fact that now magnetization is not a conservation quantity.

All listed mechanisms of longitudinal spin excitations remain valid in the high-loops approximation, too. Also, as in the case of a ferromagnet, there is a strong renormalization of the longitudinal spin excitations frequency. As a result, the energy of longitudinal spin vibration strongly differs form a simple algebraic sum of acoustic and/or exchange transverse modes energy. The analysis reveals [37] that in the $(\mathbf{q}, \omega)$ region where the first two channels are actual, the function $\chi^{z z}(\mathbf{q}, \omega)$ possesses of two types of resonances. There is a peak which characterizes a precession-like motion with the frequency $\pm \Omega_{\text {res }} \sim D q$ and damping $\gamma(\mathbf{q}, \omega) \sim D q$; both these functions linearly depend on the wave vector (the acoustic branch of longitudinal spin excitations). I.e., in the system the wave-like vibrations of spins longitudinal components exist, though with a strong attenuation. At the same time, there is also a quasi-diffusive pole at $\pm i \Omega_{\text {dif }}$, i.e., there is the quasi-relaxation mode connected with diffusion of longitudinal fluctuations, which forms the central peak in the spectral function $\omega^{-1} \operatorname{Im} \chi^{z Z}(\mathbf{q}, \omega)$. Thus, in this frequency and wave vector domains the ferrimagnet spectral function behavior is similar to that of a ferromagnet [32].

In the $(\mathbf{q}, \omega)$ region where the third channel (creation/annihilation of one acoustic and one exchange transverse modes) is actual, the leading part of the longitudinal spin susceptibility reads

$$
\chi^{z z}(\mathbf{q}, \omega) \sim \frac{\left(b_{1}-b_{2}\right)^{3}}{2 b_{1}^{2} b_{2}^{2}} \frac{J_{0}}{J_{\mathbf{q}}} \frac{N(\mathbf{q}, \omega)}{ \pm \omega+\Omega_{\mathrm{exc}}+i \gamma_{\mathrm{exc}}} .
$$

The quantity $\Omega_{\mathrm{exc}}=\Omega_{\mathrm{exc}}(\mathbf{q}, T)$ is the frequency of collective (exchange type) vibrations of longitudinal magnetization components which in this $(\mathbf{q}, \omega)$ region is described by the following expression [37]:

$$
\Omega_{\mathrm{exc}}(\mathbf{q}, T) \approx\left(b_{1}-b_{2}\right) J_{0}\left[1+2 \ln \frac{T}{\left(b_{1}-b_{2}\right) J_{0}}+\frac{\pi^{2}}{2}+f(T) q\right],
$$

where

$$
f(T) \approx 4 \frac{J_{0}^{2}}{T J_{q}}+\ln \left[\frac{\left(b_{1}-b_{2}\right) J_{0}+D}{T}\right]-2\left(1+\frac{3 D}{4 T}\right) .
$$

The exchange longitudinal mode is energetically above the exchange mode of the transverse spin-waves (14) at the same temperature and wave vector, and linearly depends on $\mathbf{q}$. The ratio $\gamma\left(\mathbf{q}, \Omega_{\text {exc }}\right) / \Omega_{\text {exc }}(\mathbf{q}, T)$ is approximately

$$
\gamma\left(\mathbf{q}, \Omega_{\mathrm{exc}}\right) / \Omega_{\mathrm{exc}}(\mathbf{q}, T) \sim \frac{b_{1} b_{2}}{b_{1}-b_{2}} \frac{J_{0}}{T}
$$

and at low temperatures $T<J_{0}$ is large enough. But at $T \sim T_{C} b_{1}<<S_{1}, b_{2}<<S_{2}$ and $\gamma\left(\mathbf{q}, \Omega_{\text {exc }}\right) / \Omega_{\text {exc }}(\mathbf{q}, T)<<1$. Thus, in a ferrimagnet wave-like excitations of longitudinal components of magnetization exist, though with a strong attenuation at low temperatures.

These results are applicable for a system with antiparallel sublattice alignment. As is known, the ground state of a ferrimagnet can be changed by strong enough magnetic field and by temperature [38]. If external magnetic field reaches some critical value $H_{s f}$, a ferrimagnet undergoes a spin-flop transition from antiparallel sublattice order to noncollinear one (the spin-flop state). The canting angles between the magnetic sublattices can be further controlled by the magnetic field. Increasing the magnetic field beyond $H_{s f}$ both sublattice magnetizations will tilt towards the external magnetic field. When the saturation field $H_{S}$ has to overcome the inter-sublattice exchange field both magnetizations align collinearly along the external field (the spin-flip state). In addition, if the larger (at $T=0$ ) sublattice magnetization decreases faster than the smaller one with growing temperature, then at certain temperature the total magnetization can become equal to zero. That is the so-called magnetic compensation point $T_{M}$. If the sublattices $g$ factors differ, $g_{1} \neq g_{2}$, the mechanical (angular momentum) compensation point $T_{L}$ does not coincide with the magnetic compensation point $T_{M}$.

The high-field ultrafast laser spectroscopy has just only started to investigate of such type noncollinear ferrimagnetic structures. Recent experimental investigations [39] reveal a strong impact of the magnetic field on the laserinduced magnetization dynamics in the ferrimagnetic alloy GdFeCo. Upon reaching the spin-flop field, the laser-induced magnetization excitations change in amplitude and timescale. The magnetization dynamics in this high-field 
regime is shown to be gradually suppressed in comparison with the antiparallel sublattice alignment.

Theoretically, features of transverse magnetization dynamics for a two-sublattice ferrimagnet near the compensation points $T_{M}$ and $T_{L}$ have been recently discussed in Ref. 40 . Yet, the longitudinal energy spectrum and susceptibility of a ferrimagnet near the compensation points, in a spin-flop phase, and for the parallel sublattice alignment state, to our knowledge, has not been still analyzed in details even for the equilibrium state of the system.

\section{Antiferromagnet}

Antiferromagnetic compounds are also promising candidates for extremely rapid spin manipulation and switching since, unlike ferromagnets, for this type of magnetic order there is no requirement of the angular momentum conservation. Even in the equilibrium state the dynamical properties of antiferromagnet essentially differ from those of ferro- and ferrimagnets. Existing experimental results [41-44] point that the ultrafast response of an antiferromagnetic system to intense optical excitation is also distinctly different from that of a ferromagnetic system.

It should be noted, however, that up to our knowledge, the longitudinal dynamics for an antiferromagnet has not been still investigated theoretically in such details as for a ferromagnet or a ferrimagnet. Here a few characteristic features should be taken into account: a total macroscopic magnetization is zero, the anisotropy plays an important role, and interaction of spin-waves in the longitudinal dynamics must be taken into account. In general, as in the case of a ferrimagnet, there are two processes that determine the longitudinal energy spectrum and susceptibility function: (i) absorption of one magnon and excitation of another one, and (ii) two-magnon excitation (absorption) [45-48]. Taking into account that the overall scattering weight of the simultaneous creation/annihilation of two magnons relative to one-magnon processes is related to the relative strength of the zero-point longitudinal quantum fluctuations in the ground state, which reduces the amount of the ordered spin moment compared to the full spin value $S$, it is expected the two-magnon channel has to be the strongest for antiferromagnets.

The dynamics of the longitudinal spin components in the isotropic quasi-2D antiferromagnet near and below the critical temperature $T<T_{N}$ ( $T_{N}$ is the Néel temperature) has been investigated thoroughly theoretically in Ref. 48 using renormalization group methods. It was predicted that the longitudinal spin components behave rather differently from transverse spin fluctuations, and while crossing over from the hydrodynamical to the critical regime, the coefficient of a spin diffusion becomes divergent. The authors supposed that such a behavior for $T<T_{N}$ could be described by taking into account all loop diagrams built from transverse spin GFs. Regarding the ferro- and ferrimagnetic case, it is reasonable to expect that summation of just this kind of diagrams is of vital importance to properly describe the longitudinal spin dynamics. Yet, as already mentioned, an in-depth analysis of the longitudinal spin dynamics in antiferromagnetic compounds within the microscopic spin GF approach is still not done.

Longitudinal spin fluctuations in the antiferromagnets experimentally was extensively studied by polarized neutron scattering $[46,47,49]$. In experiments Ref. 46 using polarization analysis, the spectra of transverse and longitudinal magnetic vibrations in the anisotropic antiferromagnet $\mathrm{MnF}_{2}$ have been separated. It was found that indeed while transverse modes are related to single-magnon scattering, the longitudinal part is essentially due to two-magnon scattering. The dynamic magnetic response due to the two-magnon creation or annihilation is separated by a gap centered near the spin-wave frequency from the central peak corresponding to neutron-magnon scattering (creation of one magnon and annihilation of another). The longitudinal energy spectrum extends to about twice the frequency of the zone boundary modes. The observed longitudinal spectra are in qualitative agreement with the theory for two-magnon processes. The neutron scattering cross section below the Néel temperature in $\mathrm{RbMnF}_{3}$ has been studied in Ref. 49 with the aid of neutron spin polarization analysis. In addition to the spin-wave scattering, a small central component was observed and found to be longitudinal in character. This longitudinal scattering is quasi-elastic, with an intensity that decreases with increasing wave vector and with decreasing temperature below $T_{N}$.

\section{Concluding remarks}

Our understanding of spin dynamics in solid-state systems is largely based on experimental and theoretical results for the equilibrium states, when the timescale of dynamical process is determined by the resonance frequency of the underlying excitation. Laser-induced femtosecond magnetism opens a new frontier for magnetic dynamics by exploring new regimes of the magnetic resonances in outof-equilibrium state. But probing such a fast magnetization change is a big challenge for experiment and theory, and complete microscopic understanding of magnetization dynamics that involves correlated interactions of spins, electrons, photons, and phonons on femtosecond timescales has yet to be developed. Apart from the complexity of the problem itself, among the reasons for the lack of fundamental understanding of ultrafast magnetism at the microscopic scale is the absence of the answer to the question how intrinsic magnetic properties can control the ultrafast dynamics of spin subsystems in magnetic materials. In other words, can ultrafast magnetization dynamics be primarily determined by the intrinsic magnetic properties of the material rather than the result of laser-induced ultrafast transient changes in the material, e.g., hot electrons or 
phonons? Naturally, the answer to this question requires understanding the role of different magnetic interactions in the ultrafast demagnetization process, and, first of all, the role of such fundamental quantum mechanical magnetic interaction as the exchange interaction. That is why, as was noted in Introduction, the author consciously restricted himself by consideration of the basic microscopic models of magneto-dielectrics - the Heisenberg model — and has not discussed roles of other subsystems (electrons, phonons, etc.) in these processes. (The reader interested in these questions is referred to reports [50-56] and references therein.)

At present, it is accepted that within the Heisenberg model approach, the dynamics of longitudinal spin components implies a different physical picture than transverse spin oscillations and reflects both the specifics of a magnetic system dynamic variables - the spin operators and the character of the magnetic order. It is ascertained that the longitudinal magnetization dynamics realizes, in general case, through three different channels: (i) a process of creation and annihilation of in-phase sublattice magnetization precessions, (ii) a process of creation and annihilation of out-of-phase sublattice magnetization precessions, and (iii) by a creation/annihilation of one in-phase and one out-of-phase sublattice magnetization excitations. The first two channels are controlled by the occupation factors determined by the Bose distribution function and provide zero contribution into the uniform susceptibility. The third channel remains in force even in the absence of thermal spin-waves excitations and makes finite the uniform susceptibility $\chi^{Z Z}(\mathbf{0}, \omega)$. Collective vibrations of magnetization longitudinal components energetically are above the transverse spin-waves frequency at the same temperature and wave vector. These results have direct relation to a final phase of the equilibration processes when after a nanosecond evolution magnetization reproduces longitudinal dynamics of the equilibrium system.

\section{Acknowledgments}

The author dedicates this article to the memory of Anatolii Illarionovich Zvyagin; in 1984-1990 I had the opportunity to participate in the work of scientific group led by A.I. Zvyagin on searching and investigating new types of magnetic structures and resonances in low-dimensional multiple sublattice antiferromagnets. The author is grateful for valuable discussions with V.G. Bar'yakhtar and B.A. Ivanov; I also extend appreciation to M. Belogolovskii for reading the manuscript and critical remarks. This work is partly supported by the Ukrainian State Fund for Fundamental Research (project No F71/59-2017 "Multifunctional Photonic Structures") and the European Union's Horizon 2020 research and innovation program under Marie Skłodowska-Curie (project "MagIC", Grant Agreement No. 644348).
1. E. Beaurepaire, J.-C. Merle, A. Daunois, and J.-Y. Bigot, Phys. Rev. Lett. 76, 4250 (1996).

2. C.D. Stanciu, F. Hansteen, A.V. Kimel, A. Kirilyuk, A. Tsukamoto, A. Itoh, and Th. Rasing, Phys. Rev. Lett. 99, 047601 (2007).

3. A.R. Khorsand, M. Savoini, A. Kirilyuk, A.V. Kimel, A. Tsukamoto, A. Itoh, and Th. Rasing, Phys. Rev. Lett. 108, 127205 (2012).

4. T. Ostler, J. Barker, R. Evans, R. Chantrell, U. Atxitia, O. Chubykalo-Fesenko, S. El Moussaoui, L. Le Guyader, E. Mengotti, L. Heyderman, F. Nolting, A. Tsukamoto, A. Itoh, D. Afanasiev, B. Ivanov, A. Kalashnikova, K. Vahaplar, J. Mentink, A. Kirilyuk, Th. Rasing, and A. Kimel, Nature Commun. 3, 666 (2012).

5. C.-H. Lambert, S. Mangin, B.S.D.C.S. Varaprasad, Y.K. Takahashi, M. Hehn, M. Cinchetti, G. Malinowski, K. Hono, Y. Fainman, M. Aeschlimann, and E.E. Fullerton, Science 345, 1337 (2014).

6. S. Mangin, M. Gottwald, C.-H. Lambert, D. Steil, V. Uhl, L. Pang, M. Hehn, S. Alebrand, M. Cinchetti, G. Malinowski, Y. Fainman, M. Aeschlimann, and E.E. Fullerton, Nat. Mater. 13, 286 (2014).

7. G. Zhang, W. Hübner, E. Beaurepaire, and J.-Y. Bigot, Topics Appl. Phys. 83, 245 (2002); In: Spin Dynamics in Confined Magnetic Structures I, B. Hillebrands and K. Ounadjela (eds.), Springer-Verlag, Berlin, Heidelberg (2002).

8. A. Kirilyuk, A.V. Kimel, and T. Rasing, Rev. Mod. Phys. 82, 2731 (2010).

9. B. Koopmans, G. Malinowski, F. Dalla Longa, D. Steiauf, M. Fähnle, T. Roth, M. Cinchetti, and M. Aeschlimann, Nat. Mater. 9, 259 (2010).

10. M. Fähnle and C. Illg, J. Phys.: Condens. Matter 23, 493201 (2011).

11. A. Kirilyuk, A.V. Kimel, and T. Rasing, Rep. Prog. Phys. 76, 026501 (2013).

12. B.Y. Mueller, T. Roth, M. Cinchetti, M. Aeschlimann, and B. Rethfeld, New J. Phys. 13, 123010 (2011).

13. S. Mathias, Chan La-O-Vorakia, P. Grychtol, P. Granitzka, E. Turgut, J.M. Shaw, R. Adam, H.T. Nembach, M.E. Siemens, S. Eich, C.M. Schneider, T.J. Silva, M. Aeschlimann, M.M. Murnane, and H.C. Kapteyn, PNAS 109, 4792 (2012).

14. I.L.M. Locht, I. Di Marco, S. Garnerone, A. Delin, and M. Battiato, Phys. Rev. B 92, 064403 (2015).

15. U. Atxitia, O. Chubykalo-Fesenko, J. Walowski, A. Mann, and M. Münzenberg, Phys. Rev. B 81, 174401 (2010).

16. U. Atxitia, P. Nieves, and O. Chubykalo-Fesenko, Phys. Rev. B 86, 104414 (2012).

17. J.H. Mentink, J. Hellsvik, D.V. Afanasiev, B.A. Ivanov, A. Kirilyuk, A.V. Kimel, O. Eriksson, M.I. Katsnelson, and Th. Rasing, Phys. Rev. Lett. 108, 057202 (2012).

18. V.G. Bar'yakhtar, V.I. Butrim, and B.A. Ivanov, JETP Lett. 98, 289 (2013).

19. U. Atxitia, T. Ostler, J. Barker, R.F.L. Evans, R.W. Chantrell, and O. Chubykalo-Fesenko, Phys. Rev. B 87, 224417 (2013).

20. S. Wienholdt, D. Hinzke, K. Carva, P.M. Oppeneer, and U. Nowak, Phys. Rev. B 88, 020406(R) (2013). 
21. I.A. Yastremsky, P.M. Oppeneer, and B.A. Ivanov, Phys. Rev. B 90, 024409 (2014).

22. W. Heisenberg, Z. Physik 49, 619 (1928).

23. Yu.A. Izyumov, F.A. Kassan-Ogly, and Yu.N. Scryabin, Field Methods in Theory of Ferromagnetism, Fiz.-Mat. Lit., Moscow (1974) [in Russian].

24. V.G. Baryakhtar, V.N. Krivoruchko, and D.A. Yablonskii, Green Functions in the Theory of Magnetism, Naukova Dumka, Kiev (1984) [in Russian].

25. Yu.A. Izyumov and Yu.N. Scryabin, Statistical Mechanics of Magnetically Ordered Systems, Consultants Bureau, New York (1988).

26. In accordance with the rules for the diagram techniques, a block encompassing $N$ operators $S^{z}$ is to be compared to the $(N-1)$ th derivative of the function $b(z)$ of the form $\sim \delta \omega_{n, 0} b^{(N-1)}(z)$, where $b(z)=S B_{S}(z)$, and $B_{S}(z)$ stands for the Brillouin function (see Refs. 23, 24 for more details).

27. R.N. Wilcox, Phys. Rev. 174, 624 (1968).

28. P.C. Kwok and T.D. Schultz, J. Phys. C 2, 1196 (1969).

29. R. Pirc and B.G. Dick, Phys. Rev. B 9, 2701 (1974).

30. D. Forster, Hydrodynamic Fluctuations, Broken Symmetry and Correlation Functions, W.A. Benjamin, Massachusetts (1975).

31. V.G. Vaks, A.I. Larkin, and S.A. Pikin, Zh. Eksp. Teor. Phys. 53, 1089 (1967) [Sov. Phys. JETP 26, 647 (1968)].

32. Yu.A. Izyumov, N.I. Chaschin, and V.Yu. Yushankhai, Phys. Rev. B 65, 214425 (2002).

33. B.P. Toperverg and A.G. Yashenkin, Phys. Rev. B 48, 16505 (1993).

34. I.D. Luzyanin, A.G. Yashenkin, S.V. Maleyev, E.A. Zaitseva, and V.P. Khavronin, Physica B 284-288, 1521 (2000).

35. Yu.G. Rudoy, Ukr. J. Phys. 50, 770 (2005).

36. S.A. Pikin, Zh. Eksp. Teor. Phys. 54, 1851 (1968) [Sov. Phys. JETP 27, 995 (1968)].

37. V.N. Krivoruchko, Phys. Rev. B 94, 054434 (2016).

38. A.G. Gurevich and G.A. Melkov, Magnetization, Oscillations and Waves, CRC Press (1996).

39. J. Becker, A. Tsukamoto, A. Kirilyuk, J.C. Maan, Th. Rasing, P.C.M. Christianen, and A.V. Kimel, Phys. Rev. Lett. 118, 117203 (2017).

40. V.N. Krivoruchko, Fiz. Nizk. Temp. 40, 55 (2014) [Low Temp. Phys. 40, 42 (2014)].
41. M. Fiebig, N.P. Duong, T. Satoh, B.B. van Aken, K. Miyano, Y. Tomioka, and Y. Tokura, J. Phys. D: Appl. Phys. 41, 164005 (2008).

42. T. Kampfrath, A. Sell, G. Klatt, A. Pashkin, S. Mährlein, T. Dekorsy, M. Wolf, M. Fiebig, A. Leitenstorfer, and R. Huber, Nat. Photon. 5, 31 (2010).

43. G. Batignani, D. Bossini, N. Di Palo, C. Ferrante, E. Pontecorvo, G. Cerullo, A. Kimel, and T. Scopigno, Nat. Photon. 9, 506 (2015).

44. D. Bossini, S. Dal Conte, Y. Hashimoto, A. Secchi, R.V. Pisarev, Th. Rasing, G. Cerullo, and A.V. Kimel, Nat. Commun. 7, 10645 (2016).

45. S. Braune and S.V. Maleyev, Z. Phys. B 81, 69 (1990).

46. W. Schweika, S.V. Maleyev, Th. Brückel, V.P. Plakhty, and L.-P. Regnault, Europhys. Lett. 60, 446 (2002).

47. I.U. Huberman, T. Coldea, R. Cowley, R.A. Tennant, D.A. Leheny, R.L. Christianson, J. Rebecca, and C.D. Frost, Phys. Rev. B 72, 014413 (2005).

48. F. Mazenko, M.J. Nolan, and R. Freedman, Phys. Rev. B 18, 2281 (1978).

49. U.J. Cox, R.A. Cowley, S. Bates, and L.D. Cussen, J. Phys.: Condens. Matter I, 3031 (1989).

50. C. Stamm, T. Kachel, N. Pontius, R. Mitzner, T. Quast, K. Holldack, S. Khan, C. Lupulescu, E.F. Aziz, M. Wietstruk, H.A. Dürr, and W. Eberhardt, Nat. Mater. 6, 740 (2007).

51. K. Carva, M. Battiato, and P. Oppeneer, Phys. Rev. Lett. 107, 207201 (2011).

52. B.Y. Mueller, T. Roth, M. Cinchetti, M. Aeschlimann, and B. Rethfeld, New J. Phys. 13, 123010 (2011).

53. T. Roth, A.J. Schellekens, S. Alebrand, O. Schmitt, D. Steil, B. Koopmans, M. Cinchetti, and M. Aeschlimann, Phys. Rev. X 2, 021006 (2012).

54. E. Turgut, C. La-o-vorakiat, J.M. Shaw, P. Grychtol, H.T. Nembach, D. Rudolf, R. Adam, M. Aeschlimann, C.M. Schneider, T.J. Silva, M.M. Murnane, H.C. Kapteyn, S. and Mathias, Phys. Rev. Lett. 110, 197201 (2013).

55. M. Haag, C. Illg, and M. Fähnle, Phys. Rev. B 90, 014417 (2014).

56. E. Turgut, D. Zusin, D. Legut, K. Carva, R. Knut, J.M. Shaw, C. Chen, Z. Tao, H.T. Nembach, T.J. Silva, S. Mathias, M. Aeschlimann, P.M. Oppeneer, H.C. Kapteyn, M.M. Murnane, and P. Grychtol, Phys. Rev. B 94, 220408(R) (2016). 